\title{
The treatment of acute myeloid leukaemia
}

\author{
J. H. FRIEND, C. GILES, AND S. G. N. RICHARDSON \\ From the Haematology Department, North Staffordshire Hospital Centre, Stoke-on-Trent
}

SYNOPSIS The use of a combination of daunorubicin and cytosine arabinoside in the treatment of acute myeloid leukaemia in a district general hospital is described. The results in a series of 30 cases are considered.

The treatment of acute myeloid leukaemia with daunorubicin (Bernard, Jacquillat, Boiron, Najean, Seligmann, Tanzer, Weil, and Lortholary, 1967), cytosine arabinoside (Henderson, Serpick, Leventhal, and Henry, 1968), and with a combination of these drugs (Crowther, Bateman, Vartan, Whitehouse, Malpas, Fairley, and Scott, 1970) produces a substantial remission rate. We decided to administer the chemotherapy described by Crowther and his colleagues (1970) in this district general hospital where acute leukaemia is one of the commoner blood diseases. This article is a record of our experience using this therapy during the first two years.

\section{Methods}

The diagnosis was based on the peripheral blood picture in all but three patients who presented with pancytopenia but was confirmed in all cases by examination of marrow films and sections. Blood and platelet counts were performed by automated methods. Blood and marrow films were stained with Leishman-Giemsa and by standard cytochemical techniques.

Treatment was given in a cycle of five days' therapy alternating with five days' rest. Daunorubicin $(1.5 \mathrm{mg} / \mathrm{kg})$ was given on the first day only and cytosine arabinoside $(2.0 \mathrm{mg} / \mathrm{kg})$ on each of the five days. Blood transfusions were given to maintain the haemoglobin above $7 \mathrm{~g} / 100 \mathrm{ml}$ and antibiotics were used whenever the temperature was raised.

\section{Results}

The results are set out in the table. During the period December 1970 to January 1973 a total of 39 cases of acute myeloid leukaemia were diagnosed in this laboratory. Twenty-seven were classified as myeloblastic, three as mainly promyelocytic, five as Received for publication 23 October 1973.

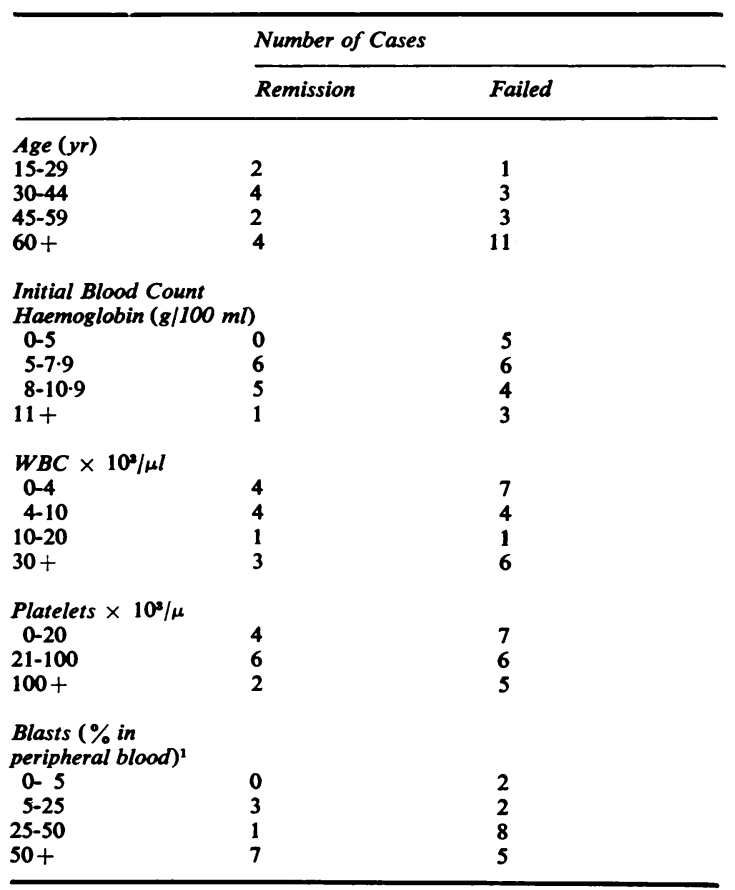

Table Analysis of factors influencing outcome of treatment

${ }^{1}$ Not recorded in two cases with profound pancytopenia.

myelo-monocytic, three undifferentiated and one as erythro-leukaemia.

Thirty-seven of these patients were admitted to hospital. Seven patients were not given specific treatment. They were too ill to allow intensive therapy and all died shortly after diagnosis (survival three-14 days, average nine days). Their ages ranged from 23 to 78 years (average 60 years).

Complete remission of the leukaemia (as defined 53 
Wrigley, Malpas, Fairley, and Scott, 1973) was obtained in 12 of the 30 cases treated with daunorubicin and cytosine (40\% remission rate). Excluding the eight patients who died during the first month of treatment the remission rate becomes $55 \%$. The successful cases received from two to five courses of treatment (average three courses) and their remissions lasted between 10 weeks and over two years (average 21 weeks). The $50 \%$ survival time was 25 weeks.

Maintenance therapy with methotrexate and 6mercaptopurine was not used regularly. In some of the earlier cases in which it was employed it appeared to have adverse effects. Later in this work we did not use maintenance therapy. There was no obvious effect on the duration of remission.

The quality of life during remission was judged crudely as good, fair, or bad. Nine patients enjoyed full health and activity during remission until relapse, one was in only fair health, whilst two patients were ill throughout remission.

Two patients obtained second remissions from the use of the same treatment, though only briefly. One patient's illness ended with intracranial involvement by the leukaemia which was undifferentiated.

Of those patients who failed to respond, eight died on average 20 days after diagnosis, having received only one course of treatment. Six patients received two or more courses and survived on average 47 days from diagnosis. Two patients lived for more than one year without treatment beyond the original attempt to induce remission.

\section{Discussion}

We have treated acute myeloid leukaemia in a district general hospital in circumstances far from ideal. Our patients were housed in open general medical wards without special attempts to prevent infection.

Apart from the two patients who were not admitted to hospital, we accepted all the cases occurring in this district and we believe that they give a realistic picture of the presentation and incidence of the disease. Seven patients appeared to be beyond aid and we did not attempt to treat them. Now, with greater experience of this therapy, we would do so, especially since treatment in their own district hospital offers the only chance to patients who are too ill to be transported any great distance.

Our failures may have been caused by delay in initiating therapy, a disinclination to persevere with this treatment in the face of increasing illness, andan inability to combat infection. In some cases these problems can be overcome but in others they still seem insuperable.

Although our criteria for inclusion in the trial were not strictly comparable with those of Crowther et al (1970), the remission rate and the duration of remissions obtained do not differ significantly from those reported by them.

We could not predict the likelihood of obtaining remission when treatment began. There was no significant correlation between the remission rate and the patient's age, nor did the initial blood count or the type of leukaemia serve as prognostic criteria. Although remission occurred in only $26.5 \%$ of patients aged 60 years or more, opposed to $53 \%$ remissions below this age, the small number of cases make this difference insignificant. We were especially disappointed by the poor response in our younger patients.

This inability to forecast accurately the result of treatment meant that many of our patients received treatment which not only proved ineffective but subjected them to discomfort and distress in their terminal illness.

No patients have been classified as partial remissions. Many of those in whom therapy failed showed a haematological response during treatment in that the marrow blast population was suppressed but this was not maintained for more than a few days. Two patients, however, survived for 15 months and more than 12 months respectively in comparatively good health though obviously not in remission with virtually unchanged blood and marrow counts. We cannot regard these cases in any way as therapeutic successes and doubt that their disease was modified by the attempts to induce remission. Before effective treatment was available occasional cases of acute leukaemia survived for long periods.

Whilst these are better results than any obtained with previous treatment regimes they give little satisfaction and emphasize the continuing need for improved techniques and more effective drugs. Such progress will demand large-scale controlled trials. We believe, however, that acute myeloid leukaemia is sufficiently common to justify the application of proven therapy in district hospitals.

\section{References}

Bernard, J., Jacquillat, C., Boiron, M., Najean, I., Seligmann, M., Tanzer, J., Weil, M., and Lortholary, P. (1967). Essai de traitement des leucémies aigués lymphoblastiques et myéloblastiques par un antibiotique nouveau: la rubidomycine (13057 RP) Etude de 61 observations. Presse méd., 75, 951-955.

Crowther, D., Bateman C. J. T., Vartan, C. P., Whitehouse, J. M. A., Malpas, J. S., Fairley, G. H., and Scott, Sir R. B. (1)70). Combination chemotherapy using L-asparaginase, datnorubicin and cytosine arabinoside in adults with acute myclogenous leukaemia. Brit. med. J., 4, 513-517.

Crowther, D., Powles, R. L., Bateman, C. J. T., Beard, M. E. J., Gauci, C. L., Wrigley, P. F. M., Malpas, J. S., Fairley, G. H.. and Scott, Sir R. B. (1973). Management of adult acute myelogenous leukaemia. Brit. med. J., 1, 131-137.

Henderson, E., Serpick A., Leventhal, B., and Henry, P. (1969). Cytosine arabinoside infusions in adult and childhood myelocytic leukaemia. Proc. Amer. Ass. Cancer Res., 9, 29. 